\title{
Silicon Photonics for On-Chip Spectrophotometry
}

\author{
Danaë Delbeke, Ananth Z. Subramanian, Paolo Cardile, Wouter Woestenborghs, Alfonso Ruocco, Jan-Willem Hoste, \\ Daan Martens, Ashim Dhakal, Peter Bienstman, Gunther Roelkens, Nicolas Le Thomas, Wim Bogaerts, Roel Baets \\ Photonics Research Group, INTEC-department, Ghent University - imec \\ Center for Nano- and Biophotonics, Ghent University \\ Sint-Pietersnieuwstraat 41, B-9000 Ghent, Belgium
}

(invited)

\begin{abstract}
Silicon and Silicon Nitride photonics are on their way to open the route towards integrated on-chip spectrophotometers. Cost, manufacturability, miniaturization, but also performance advantages are at the origin of their potential. We will discuss several integrated on-chip spectrophotometers that are on the eve of commercial take up.
\end{abstract}

\section{Keywords—silicon photonics; on-chip spectrophotometer}

\section{INTRODUCTION}

Spectrophotometry is the quantitative measurement of the reflection or transmission properties of a material, an object or substance as a function of wavelength. A spectrophotometer measures the intensity of the light as a function of its wavelength. Spectrometers have become the most important analytical instrument in today's laboratory for the spectroscopic detection of a wide variety of atoms and molecules in applications ranging from physics and chemistry to biology and environmental sciences. Besides spectroscopic chemical and biochemical sensing applications, spectrometers are also used in a wide variety of applications for which the response is measured by a spectral shift. Examples are fiber bragg grating (FBG) sensor interrogators, spectral-domain optical coherency tomography (OCT) and resonator sensors.

For these applications, the user is in search for ever smaller, lower cost, extremely ruggedized spectrometers. The need for data captured in the field and the ubiquitous use of sensors for in-situ monitoring of systems and environments are driving this demand. Integrated photonics, and in particular Silicon On Insulator (SOI) technology offers an opportunity for lowcost, mass manufactured, miniaturized, monolithic optical systems. This technology makes use of the technologies available in an advanced CMOS fab to fabricate photonic integrated circuits on 200 or $300 \mathrm{~mm}$ SOI wafers [1-3]. Fabrication of optical devices with the same silicon processing tools and wafers that the semiconductor industry uses to fabricate CMOS transistors creates access to an immense infrastructure for yield improvement, metrology and process control. By making use of a silicon-nitride $\left(\mathrm{Si}_{3} \mathrm{~N}_{4}\right)$ waveguide layer, the technology can be extended to the entire visible and near IR wavelength range [4]. Mid IR wavelengths can be addressed by the replacement of silicon-oxide by a cladding material that is transparent in the mid IR [5].

Silicon photonics has benefitted from the multi-billion investments spent during the last decades in CMOS fabs. The use of the established CMOS infrastructure for optical systems allows for a high level of integration, high process control and yield and might introduce the economy of scale. This is in contrast to the current state of optical systems, where different technologies are used to realise the respective optical functionalities all interconnected via lenses or optical fibers.

The downside of exploiting the CMOS infrastructure is the cost of accessing it. Typically, a dedicated run in a process that yields waveguides, modulators and photodetectors will cost a couple of hundreds of thousands of euros. If dedicated process steps need to be developed, the cost is even higher. Multi Project Wafer (MPW) platforms have been set-up such as ePIXfab/Europractice that offer a cost-sharing access to readily developed processes with various levels of capability for prototyping. In spite of these MPW platforms that reduce substantially the financial risk for prototyping and pave the route for scaling to production, the volumes of photonic nondata/telecom applications in general and spectrometer applications specifically is too low to surf on the economy of scales that is typically claimed to be one of the main drivers for silicon photonics. Silicon photonics is still an expensive industry to start up activities, while the cost for small-tomedium volumes of devices tends to the cost of existing products. On top of this, looking at miniaturization, state-ofthe-art spectrometers using discrete optical components have themselves enjoyed a trend towards miniaturized devices. Handheld spectrometers using classical optical components, connected with lenses and fibers, are state-of-the art.

For start-up commercial activities in the short term, the returnon-investment (ROI) considering the development of silicon photonics based spectrometers cannot compete with existing products on cost figure of merits only. Silicon photonics will only be the technology of choice with the potential of a positive ROI for these applications for which silicon photonics brings in that unique enabler not possible to realise with discrete optics:

1. Applications that need an extreme miniaturization down to the level of smart grains; the high refractive index and monolithic integration of silicon photonics is the unique selling proposition (USP)

2. Applications that need extreme ruggedness; the monolithic integration of optical functionalization on silicon photonics chips introduce the ruggedness specific to micro-electronics into optical systems. 
3. Applications that need a high optical complexity (integration of many optical functions or massive parallelism); the cost of optical systems dominated by packaging is alleviated by integrating many optical functions in a single package. In this case, cost will be the USP after all, even for small-to-medium volumes.

4. Applications for which photonic integration in high index contrast circuits results in an increased performance.

In section II we describe 4 applications that require one or more of these silicon photonics USPs, opening a route towards commercial take-up of silicon photonics for spectrophotometry in the short term, because

\section{SPECTROMETER APPLICATIONS EXPLOITING SILICON PHOTONICS USP}

\section{A. Continuous glucose monitoring sensor exploiting silicon photonics' asset of extreme miniaturization}

Continuous glucose monitoring (CGM) systems today use a tiny chemical sensor inserted under the skin to check glucose levels in tissue fluid day and night. CGM offers patients a better and safer glycemic control. Many research efforts have shown that it is possible to extract the glucose signature from near-infrared (NIR) absorption spectra [6]. This detection method avoids the use of reagents to ensure long-term sensor reliability and additionally, allows the detection of multiple bio-molecules apart from glucose. An implantable CGM device circumvents the variability of the skin, but requires a grain sized spectrometer. An implantable CGM device based in an integrated silicon photonics spectrometer was proposed earlier, and exploits the unique asset of extreme miniaturization of silicon photonics [6].

\section{B. In-situ operational structural monitoring: exploiting silicon photonics' asset of extreme ruggedness}

Partially or fully instrumented structures give feedback on their structural response to any applied loading condition. As such the operator or inspector is able to determine if operation is safe, i.e. "all in the green". Negative signals can reveal suboptimal loading or use of the structure or indicate that maintenance is necessary. This concept of a smart systems is not new and exists already more than several decades, with embedded or mounted FBG optical fiber sensors being the most popular and reliable solution. However, implementation of embedded FBG sensor technology was mostly limited to academic research and prototyping level. The lack of extremely ruggedized interrogators that can work in harsh environments being at the same time low-cost and small is retaining the actual take up of this technology by the industry for continuous in-situ operational monitoring. A silicon photonics based FBG interrogator has been developed to enable in-situ operational monitoring of systems such as turbine blades, vessels, etc. [7]. The monolithic (ruggedness) integration of all optical functionalities in a single package (alleviation of packaging cost) makes silicon photonics the preferential technology platform for this application.

\section{Low-cost, multiplexed refractive-index based biosensor diagnostic tool: exploiting silicon photonics' asset of massive parallelism an high optical complexity}

Cheap SOI refractive-index based label-free biosensors have been demonstrated that allow fast and accurate quantitative detection of biologically relevant molecules for applications in medical diagnostics. However, whereas the sensor chip can be made cheaply, an expensive tunable laser is typically required to accurately monitor spectral shifts in the sensor's transmission spectrum (wavelength interrogation). To address this issue, silicon photonics can push the cost further down by integrating more functionalities on the chip to bring the cost of packaging and read-out down [8]. By integrating with each sensor a wavelength demultiplexer that divides the sensor transmission spectrum in multiple wavelength channels and transmitting them to spatially separated output ports, wavelength interrogation with a much cheaper broadband light source is enabled. Using $\mathrm{Si}_{3} \mathrm{~N}_{4}$ [4], the read-out/packaging cost can be further decreased allowing low cost visible LEDs and ubiquitous low-cost CCD camera chips, both coupled via freespace to the chip. Several 10's of sensors, including AWG, can be multiplexed on a chip, allowing for an equal amount of detectable biomarkers. Silicon (nitiride) photonics is the enabler for a rapid, accurate diagnostic tools.

\section{Raman-on-chip: exploiting silicon photonics' asset of increased performance}

Raman spectroscopy gains importance as a label-free and foolproof detection method for a variety of chemical substances and biomolecules. The major barrier to the widespread use of Raman spectroscopy is the fact that the spontaneous Raman scattering process is extremely weak. Through the use of $\mathrm{Si}_{3} \mathrm{~N}_{4}$ waveguide we have demonstrated that the use of such single mode high contrast waveguide enhances the Raman signal by at least a factor of 500 per $\mathrm{cm}$ of waveguide length compared to the confocal microscopy system. This direct indication of performance increase of AN integrated approach in comparison with discrete optics systems puts $\mathrm{Si}_{3} \mathrm{~N}_{4}$ Raman-on-chip in a very competitive position for new Raman spectroscopy applications.

\section{REFERENCES}

[1] Baets, R., "Building a sustainable future for silicon photonics", 8th International Conference on Group IV Photonics 2011, United Kingdom, p. 3-4 (PLE2) (2011).

[2] Bogaerts, W., Selvaraja, S., Dumon, P., Brouckaert, J., De Vos, K., Van Thourhout, D. and Baets, R., "Silicon-on-Insulator Spectral Filters Fabricated with CMOS Technology", IEEE J. Sel. Top. Quantum Electronics, 16(1), p.33-44 (2010).

[3] Selvaraja, S., Bogaerts, W., Dumon, P., Van Thourhout, D. and Baets, R., Sub-nanometer linewidth uniformity in silicon nano-photonic waveguide devices using CMOS fabrication technology, IEEE Journal on Selected Topics in Quantum Electronics, 16(1), p.316 - 324 (2010).

[4] D. Martens, A. Subramanian, S. Pathak, M. Vanslembrouck, P. Bienstman, W. Bogaerts, R. Baets, Compact Silicon Nitride Arrayed Waveguide Gratings for Very Near-infrared Wavelengths, Photonics Technology Letters, (2014)

[5] E.M.P. Ryckeboer, A. Gassenq, M. Muneeb, N. Hattasan, S. Pathak, L. Cerutti, J-B Rodriguez, E. Tournie, W. Bogaerts, R. Baets, G. Roelkens, Silicon-on-insulator spectrometers with integrated GaInAsSb photodiodes for wide-band spectroscopy from 1510 to $2300 \mathrm{~nm}$, Optics Express, (2013)

[6] E.M.P. Ryckeboer, R. Bockstaele, M. Vanslembrouck, R. Baets, Glucose sensing by waveguide-based absorption spectroscopy on a silicon chip, Biomedical optics express, 5(5), p.1636-1648 (2014)

[7] A. Ruocco, D. Van Thourhout, W. Bogaerts, Silicon Photonic Spectrometer for Accurate Peak Detection using the Vernier Effect and Time-Domain Multiplexing, Journal of Lightwave Technology, p.3351 3357 (2014)

[8] T. Claes, W. Bogaerts, P. Bienstman, Vernier-cascade label-free biosensor with integrated arrayed waveguide grating for wavelength interrogation with low-cost broadband source, Optics Letters, 36(17), p.3320--3322 (2011)

[9] A. Dhakal, A. Subramanian, P.C. Wuytens, F. Peyskens, N. Le Thomas, R. Baets, Evanescent excitation and collection of spontaneous Raman spectra using silicon nitride nanophotonic waveguides, Optics Letters, 39(13), p.4025-4028 (2014) 
